${ }^{a}$ Cardiology Division,

Department of Medicine, Queen Mary Hospital, the University of Hong Kong, Hong Kong, SAR, China; 'Shenzhen Institutes of Research and Innovation, the University of Hong Kong, Hong Kong, SAR, China; 'Hong Kong-Guangdong Joint Laboratory on Stem Cell and Regenerative Medicine, The University of Hong Kong and Guangzhou Institutes of Biomedicine and Health, China; 'Laboratory of Chromatin and Human Disease, Key Laboratory of Regenerative Biology of the Chinese Academy of Sciences and Guangdong Provincial Key Laboratory of Stem Cells and Regenerative Medicine, Guangzhou Institutes of Biomedicine and Health, Chinese Academy of Sciences, Guangzhou, 510530, China; ${ }^{\mathrm{e}}$ Research Center of Heart, Brain, Hormone and Healthy Aging, Li Ka Shing Faculty of Medicine, The University of Hong Kong, Hong Kong, SAR, China

Correspondence: Hung-Fat Tse, M.D., Ph.D., Cardiology Division, Department of Medicine, The University of Hong Kong, Rm 1928, Block K, Queen Mary Hospital, Hong Kong, China. Telephone: +852 22554694; Fax: + 852 28186304; email: hftse@hkucc.hku.hk

Received February 22, 2016; accepted for publication May 28, 2016; first published online in Stem Cells Express June 22, 2016.

(c) AlphaMed Press 1066-5099/2016/\$30.00/0

http://dx.doi.org/ 10.1002/stem.2438

This is an open access article under the terms of the Creative Commons Attribution-NonCommercialNoDerivs License, which permits use and distribution in any medium, provided the original work is properly cited, the use is non-commercial and no modifications or adaptations are made.

\title{
Generation of Induced Cardiospheres via Reprogramming of Skin Fibroblasts for Myocardial Regeneration
}

\author{
JiAn-Yong Xu, ${ }^{a, b}$ YeE-Kı LeE, ${ }^{a}$ XINRU RAN, ${ }^{a}$ SONG-YAN LIAO, ${ }^{a}$ JiAYIN YANG, ${ }^{a, b}$ Ka-Wing Au, \\ Wing-Hon Lai, ${ }^{a}$ Miguel A. Esteban, ${ }^{a, c, d}$ Hung-Fat Tse ${ }^{a, b, c, e}$ \\ Key Words. Cardiospheres $•$ Cardiac progenitor cells $•$ Myocardial regeneration $•$ Somatic \\ reprogramming $\cdot$ Skin fibroblast
}

\section{ABSTRACT}

Recent pre-clinical and clinical studies have suggested that endogenous cardiospheres (eCS) are potentially safe and effective for cardiac regeneration following myocardial infarction (MI). Nevertheless the preparation of autologous eCS requires invasive myocardial biopsy with limited yield. We describe a novel approach to generate induced cardiospheres (iCS) from adult skin fibroblasts via somatic reprogramming. After infection with Sox2, KIf4, and Oct4, iCS were generated from mouse adult skin fibroblasts treated with Gsk3ß inhibitor- $\left(2^{\prime} Z, 3^{\prime} E\right)-6$ Bromoindirubin- $3^{\prime}$-oxime and Oncostatin $M$. They resembled eCS, but contained a higher percentage of cells expressing Mesp1, IsI1, and Nkx2.5. They were differentiated into functional cardiomyocytes in vitro with similar electrophysiological properties, calcium transient and contractile function to eCS and mouse embryonic stem cell-derived cardiomyocytes. Transplantation of iCS $\left(1 \times 10^{6}\right.$ cells) into mouse myocardium following MI had similar effects to transplantation of eCS but significantly better than saline or fibroblast in improving left ventricular ejection fraction, increasing anterior/septal ventricular wall thickness and capillary density in the infarcted region 4 weeks after transplantation. No tumor formation was observed. iCS generated from adult skin fibroblasts by somatic reprogramming and a cocktail of Gsk3 $\beta$ inhibitor-6Bromoindirubin- $\mathbf{3}^{\prime}$-oxime and Oncostatin $\mathrm{M}$ may represent a novel source for cell therapy in MI. STEM CELLS 2016;34:2693-2706

\section{Significance Statement}

This study provides the first proof-of-principle results that demonstrate the feasibility of generating donor-specific induced cardiospheres via a somatic reprogramming process, providing a new source of cell therapy for treatment of myocardial infarction

\section{INTRODUCTION}

Cardiovascular diseases (CVDs), especially myocardial infarction (MI), are the leading cause of death worldwide and contribute to over $30 \%$ of global mortality [1, 2]. Despite recent advances in the treatment of CVDs, a significant proportion of patients still die of progressive heart failure. Therapy remains palliative and does not address the fundamental pathogenic process of CVDs, that is, loss of functional cardiomyocytes. As a result, cell-based therapies have been proposed as a promising strategy to treat CVDs via replenishment of the damaged cells. Different types of stem cells, including bone marrow stem cells, mesenchymal stem cells, skeletal myoblasts, endothelial progenitor cells and cardiac progenitor cells, have been investigated as sources for cell therapy in myocardial regeneration [3-5]. Although improved heart function has been observed in preclinical studies after transplantation of non-heart origin stem cells into the injured myocardium, there is very limited evidence of myocardial regeneration via transdifferentiation into functional cardiomyocytes in vivo. Most studies have shown that the improvement in cardiac function, especially with bone marrow-derived cells, is due to paracrine effects $[6,7]$. On the contrary, it has been demonstrated that various types of cardiac progenitor cells, including endogenous cardiospheres (eCS), can differentiate into functional cardiomyocytes in vivo, and thus may represent a more effective cell-based therapy $[8,9]$.

Cardiac progenitor cells, residing in the heart with the ability to differentiate into 
three major cardiac cell types (cardiomyocyte, endothelial cell, and smooth muscle cell), have been defined and isolated using different approaches, such as different marker-positive cell population (cKit+ [10], Flk + [11], Wt1 + [12], Mesp1+

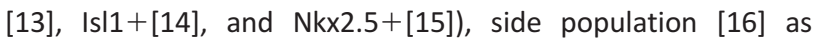
well as the eCS $[8,9]$. These cardiac progenitor cells have been consistently shown to improve heart function in different pre-clinical small and large animal models of $\mathrm{MI}[17,18]$. Among them, eCS has been one of the most commonly used cell sources for cardiac regeneration in animal models and clinical studies [18-20].

Unfortunately, the therapeutic application of autologous eCS is limited by the low yield of cell preparation as very few exist in vivo and the need for highly invasive myocardial biopsy [9]. There is thus emerging interest in the generation of cardiospheres using an alternative approach [21]. Previous reports have demonstrated that mouse embryonic fibroblasts can be directly converted into cardiomyocytes through a direct somatic reprogramming strategy; and this process passed through a cardiac progenitor stage $[22,23]$. To evaluate the feasibility of isolating these cardiac progenitor cells via the formation of cardiospheres during the somatic reprogramming process, different panels of cardiotrophic growth factors or chemicals were screened.

Our results demonstrated that the combination of Gsk3 $\beta$ inhibitor-(2'Z,3'E)- 6-Bromoindirubin-3'-oxime (BIO), and Oncostatin M (OSM) promoted the generation of spheres from both embryonic fibroblasts and adult skin fibroblasts during somatic reprogramming. These spheres, which resemble eCS, contained Mesp1, Isl1, and Nkx2.5 positive cardiac progenitor cells but not pluripotent stem cells and possessed the differentiation ability of cardiomyocytes, thus they were termed induced cardiospheres (iCS). These iCS had therapeutic potential comparable with eCS in a mouse model of $\mathrm{Ml}$. Therefore, we provide the first proof-of-principle results to show the feasibility of generating iCS for cardiac regeneration by somatic reprogramming of mouse fibroblasts without passing through a pluripotent stem cell stage by using a panel of pluripotent transcription factors and cardiotrophic growth factors.

\section{Materials AND Methods}

\section{Cell Isolation and Maintenance}

Adult skin fibroblasts were isolated from 4-week-old mice. Neonatal cardiomyocytes were isolated from 1 to 2 day old neonatal mice. Adult cardiomyocytes were isolated from 4 to 6 weeks old mice using the Langendorff retrograde perfusion method [24]. Endogenous cardiospheres were isolated as described before $[8,9]$. In brief, mouse eCS were isolated from 4 weeks old mice. The mice were anesthetized with ketamine $(100 \mathrm{mg} / \mathrm{kg})$ and xylazine $(10 \mathrm{mg} / \mathrm{kg})$, and their hearts were removed quickly and incubated with cold $\mathrm{Ca}^{2+}$ and $\mathrm{Mg}^{2+}$ free phosphate buffered saline (PBS) (Invitrogen, Grand Island, NY, USA, https://www.thermofisher.com/hk/en/ home/brands/invitrogen.html). The heart was washed with PBS via aorta perfusion, and then minced in the digestion buffer $(4 \mathrm{mg} / \mathrm{ml}$ collagenase $B$ and $4 \mathrm{mg} / \mathrm{ml}$ dispase (Roche Life Science, Penzberg, Germany, https://lifescience.roche. com/) in DMEM/High Glucose) and digested for 30 minutes at $37^{\circ} \mathrm{C}$. The isolated single cells and tissue fragments were washed twice with PBS and plated on a culture dish with culture medium (DMEM/High Glucose, 10\% FBS (fetal bovine serum), $100 \mathrm{U} / \mathrm{ml}$ penicillin, $100 \mu \mathrm{g} / \mathrm{ml}$ streptomycin, $2 \mathrm{mM} \mathrm{L-}$ glutamine, and $0.1 \mathrm{mM} \mathrm{2-mercaptoethanol)} \mathrm{at} 37^{\circ} \mathrm{C}$ and $5 \%$ $\mathrm{CO}_{2}$. Medium was refreshed every 3 days. After 3-4 weeks, the small, phase-bright cells were harvested through $0.53 \mathrm{mM}$ EDTA (Versene, Invitrogen) and $0.05 \%$ trypsin-EDTA (Invitrogen) treatment. Cells were plated onto a poly-D-lysine (Sigma, St. Louis, MO, USA, http://www.sigmaaldrich.com/) coated cell culture dish in cardiosphere-growing medium (35\% complete IMDM/65\% DMEM-Ham F-12 mix containing 2\% B27, $0.1 \mathrm{mM}$ 2-mercaptoethanol, $10 \mathrm{ng} / \mathrm{ml}$ epidermal growth factor (EGF), $20 \mathrm{ng} / \mathrm{ml}$ basic fibroblast growth factor (bFGF), $40 \mathrm{nM}$ cardiotrophin-1, $40 \mathrm{nM}$ thrombin, $100 \mathrm{U} / \mathrm{ml}$ penicillin, $100 \mu \mathrm{g} / \mathrm{ml}$ streptomycin and $2 \mathrm{mM}$ L-glutamine). Three days after plating, the eCS were formed and used for subsequent experiments.

Mouse embryonic stem cells (mESC, cell line R1) were maintained on $0.1 \%$ gelatin coated plates with culture medium (DMEM/High Glucose, 15\% FBS (HyClone, South Logan, Utah, USA, http://www.gelifesciences.com/), 2 mM GlutaMAX, $1 \%$ nonessential amino acids, $0.1 \mathrm{mM}$ 2-mercaptoethanol (Gibco, Grand Island, NY, USA, https://www.thermofisher.com/), $1 \%$ penicillin-streptomycin, and 1,000 $\mathrm{U} / \mathrm{ml}$ leukemia inhibitory factor (LIF, Millipore, Darmstadt, Germany, http://www. emdmillipore.com/). The medium was refreshed every day. Human dermal fibroblasts (Thermo Fisher, Grand Island, NY, USA, https://www.thermofisher.com/) were maintained according to manufacturer's instructions.

\section{iCS Generation and Differentiation}

Retrovirus was produced by introducing pMXs-Oct4 (Addgene 13366, Cambridge, MA, USA, https://www.addgene.org/), pMXs-Sox2 (Addgene 13367), and pMXs-Klf4 (Addgene 13370) [25] and packaging plasmid pCL-Eco (Addgene 12371) [26] into 293T cells with lipofectamine 2000 (Invitrogen). Fortyeight hours after transfection, the viral supernatant was collected and filtered using a $0.45 \mu \mathrm{m}$ PVDF-based filter for somatic cell infection. Mouse adult skin fibroblasts were plated on a $0.1 \%$ gelatin coated plate at a cell density of $2 \times 10^{4}$ cells per well. The fibroblasts were infected overnight in the presence of $8 \mu \mathrm{g} / \mathrm{ml}$ polybrene (Sigma). Then the medium was changed to basal medium: DMEM/High Glucose, 10\% Knockout Serum Replacement, 2 mM GlutaMAX, 1\% nonessential amino acids, $0.1 \mathrm{mM}$ 2-mercaptoethanol, 0.5\% N2 (Gibco) and $4 \mathrm{ng} / \mathrm{ml}$ bFGF (Peprotech, Rocky Hill, NJ, USA, https://www.peprotech.com/) for induced cardiosphere generation. Growth factors or chemicals were added at a final concentration of $20 \mathrm{ng} / \mathrm{ml}$ [EGF, bFGF, bone morphogenetic protein 4 (BMP4), Interleukin 6 from Peprotech], $50 \mathrm{ng} / \mathrm{ml}$ [vascular endothelial growth factor (VEGF), hepatocyte growth factor (HGF), and Actinin A from Peprotech; and OSM from Sigma], $100 \mathrm{ng} / \mathrm{ml}$ [Wnt3a, Cardiotrophin 1 (CT-1) from Peprotech], $20 \mathrm{mM}$ Lithium chloride (Sigma) and $2.5 \mu \mathrm{M}$ BIO (Sigma).

For knock down experiments, shRNA targeting to $\beta$ catenin (shRNA target $5^{\prime}$-GGTGCTGTCTGTCTGCTCTA-3') was cloned into lentivirus pLKO.1 puro vector with Agel and EcoRI restriction sites (Addgene 8453 ) and the infection was conducted at the same time as Oct4, Sox2, and Klf4 infection. For 
overexpression experiments, mouse $\beta$-catenin cDNA was ligated into the Sal1 and BamH1 site of the pMXs vector with infection conducted at the same time as Oct4, Sox2, and Klf4 infection.

For spontaneous differentiation, newly formed iCS were plated onto Matrigel (1:40, BD Biosciences, San Jose, CA, USA, http://www.bdbiosciences.com/) coated plates with differentiation medium (DMEM/High Glucose, 15\% FBS (HyClone), $2 \mathrm{mM}$ GlutaMAX, 1\% nonessential amino acids, $0.1 \mathrm{mM}$ 2mercaptoethanol (Gibco), $50 \mu \mathrm{g} / \mathrm{ml}$ Ascorbic acid (Sigma)) for 15 days. Beating area appeared at day 12 after differentiation and the number increased till day 15 . There was no obvious further increasing of beating area after 15 days of differentiation. This differentiation process is consistent in all experiments performed. Beating areas were manually dissected and digested with $1 \mathrm{mg} / \mathrm{ml}$ collagenase $B$ for subsequent experiments. The other differentiated cells were used for endothelial and smooth muscle cell marker staining.

Similarly, spontaneous differentiation of eCS was performed as described before $[8,9]$. The eCS were plated onto $0.1 \%$ gelatin coated plates with cardiomyocytes differentiation medium [DMEM/High Glucose, 15\% FBS (HyClone), $2 \mathrm{mM}$ GlutaMAX, 1\% nonessential amino acids, $0.1 \mathrm{mM}$ 2mercaptoethanol (Gibco), $50 \mu \mathrm{g} / \mathrm{ml}$ Ascorbic acid (Sigma)]. The beating clusters from day 15 of differentiation were manually picked out and digested with $1 \mathrm{mg} / \mathrm{ml}$ collagenase $B$ for 2 hours at $37^{\circ} \mathrm{C}$, followed by $5 \mathrm{mM}$ EDTA treatment for 5 minutes. The single cardiomyocytes were plated on $0.1 \%$ gelatin coated coverslips with cardiomyocyte differentiation medium for 3 days and then used for subsequent experiments, including flow cytometry, action potential, calcium transient, and contractility analysis.

\section{mESC Differentiation}

Cardiomyocyte differentiation of mESC was performed as described before [27]. In brief, $1 \times 10^{6}$ cells were suspended in $10 \mathrm{ml}$ differentiation medium [DMEM/High Glucose, 15\% FBS (HyClone), 2 mM GlutaMAX, 1\% nonessential amino acids, $0.1 \mathrm{mM}$ 2-mercaptoethanol (Gibco), $50 \mu \mathrm{g} / \mathrm{ml}$ Ascorbic acid (Sigma)] for 7 days to allow embryoid body (EB) formation. On day 7 , the EBs were plated onto $0.1 \%$ gelatin coated bacterial dishes for further differentiation. On day 9, beating areas were observed in some EBs. The beating cluster from day 15 of differentiation was manually picked out and digested with $1 \mathrm{mg} / \mathrm{ml}$ collagenase $B$ for 2 hours at $37^{\circ} \mathrm{C}$, followed by $5 \mathrm{mM}$ EDTA treatment for 5 minutes. The single cardiomyocytes were plated on $0.1 \%$ gelatin coated coverslips with cardiomyocyte differentiation medium for 3 days and then used for subsequent experiments.

\section{RNA Extraction and Real-Time PCR}

Cells were lysed with TRIzol Reagent (Ambion, Grand Island, NY, USA, https://www.thermofisher.com/hk/en/home/brands/ invitrogen/ambion.html). RNA was converted into cDNA with M-MuLV Reverse Transcriptase (NEW ENGLAND BioLabs, Ipswich, MA, USA, https://www.neb.com/) and random primers (Invitrogen). Real-time PCR was performed with SYBR Green Master Mix (Clontech, Mountain View, CA, USA, https://www. clontech.com/) on an ABI 7500 Fast Real-Time PCR system (Applied Biosystems, Grand Island, NY, USA, http://www.thermofisher.com/hk/en/home/brands/applied-biosystems.html) according to the manufacturer's instructions. Primers used for real-time PCR are listed in Supporting Information Table 1. qPCR determination of gene level was calculated using the $\Delta \Delta \mathrm{Ct}$ method with treatment group compared with baseline and $\beta$-actin serving as internal control.

\section{Immunofluorescence}

Cells were plated onto $0.1 \%$ gelatin coated coverslips for 48 hours, fixed with $4 \%$ paraformaldehyde (Sigma) for 10 minutes at room temperature, washed twice with PBS, permeablized with $0.2 \%$ Triton-X 100 in PBS with $10 \%$ FBS for 30 minutes, and incubated with primary antibodies (Mesp1 (Abcam, 1:100, Cambridge, UK, http://www.abcam.com/), Isl1 (Abcam, 1:100), Nkx2.5 (Santa Cruz, 1:100, Dallas, Texas, USA, http://www.scbt.com/), $\alpha$-Actinin (Sigma, 1:1,000), myosin light chain 2 atrial (Mlc2a, Abcam, 1:100), myosin light chain 2 ventricular (Mlc2v, AXXORA, 1:10, Farmingdale, NY, USA, http://www.axxora.com/), von Willebrand Factor (vWF) (Millipore, 1:100), $\alpha$-smooth muscle actin (R\&D Systems, 1:100, Minneapolis, MN, USA, https://www.rndsystems.com/), or Nanog (Fisher Scientific, 1:100, Grand Island, NY, USA, https:// www.fishersci.com/)) in blocking buffer $(0.1 \%$ Triton-X 100 in PBS with $10 \% \mathrm{FBS}$ ) overnight at $4^{\circ} \mathrm{C}$. Cells were washed five times with PBST (0.1\% Triton-X 100 in PBS), five minutes each time, and then incubated with secondary antibody Alexa Fluor 594 conjugate for 1 hour. The cells were further counterstained and mounted with SlowFade Gold antifade reagent with DAPI (Invitrogen). For counting Mesp1, Isl1, and Nkx2.5 positive cells, four views per coverslip were counted and three coverslips were used for each round of experiment. The experiment was repeated three times.

\section{Flow Cytometry and Cell Purification}

Spheres and differentiated cardiomyocytes were dissociated with $1 \mathrm{mg} / \mathrm{ml}$ collagenase B (Roche Life Science) for 30 minutes at $37^{\circ} \mathrm{C}$, followed by $2 \mathrm{mM}$ EDTA for 5 minutes. Cells were centrifuged and re-suspended in PBS with 5\% FBS. For cell surface marker staining, cells were ready for use. For cardiomyocyte marker staining, cells were fixed with $2 \%$ paraformaldehyde for 15 minutes, then permeablized with $0.5 \%$ Tween 20 for 15 minutes. Cells were stained with primary antibodies ( $\alpha$-Actinin (Sigma, 1:1,000), myosin light chain 2 atrial (Mlc2a, Abcam, 1:100), myosin light chain 2 ventricular (Mlc2v, AXXORA, 1:10), cKit (BD Biosciences, 1:100), Flk1 (BD Biosciences, 1:100), CXCR4 (Abcam, 1:100), PDGFRa (Cell signal, 1:100, Boston, MA, USA, http://www.cellsignal.com/), Sca1(Abcam, 1:100), and CD105-FITC/CD45-PE (BD Biosciences, 1:100)) for 1 hour, washed three times with PBS, then stained with secondary antibody Alexa Fluor 488 conjugate for 1 hour. Cells were analyzed with the FC500 (Beckman Coulter, Inc., Indianapolis IN, USA, https://www.beckmancoulter.com/) and data analyzed with FlowJo software. cKit, Flk1, and CXCR4 positive cells were purified by BD FACSAria SORP cell sorter (BD Biosciences).

\section{Patch Clamping}

The whole-cell patch clamp technique was used [28]. Borosilicate glass electrodes (1.2 mm outside diameter) were pulled with a Brown-Flaming puller (model P-97; Sutter Instrument Co., Novato, CA, USA, http://www.sutter.com/) with tip resistances of 2-3 $\mathrm{M} \Omega$ when filled with pipette solution $(20 \mathrm{mM}$ 
$\mathrm{KCl}, 110 \mathrm{mM} \mathrm{K}$-aspartate, $1.0 \mathrm{mM} \mathrm{MgCl}$, $10 \mathrm{mM}$ HEPES, $0.05 \mathrm{mM}$ EGTA, $0.1 \mathrm{mM}$ GTP, $5.0 \mathrm{mM}$ Na2-phosphocreatine, and $5.0 \mathrm{mM} \mathrm{Mg2-ATP}$; the $\mathrm{pH}$ was adjusted to 7.2 with $\mathrm{KOH}$.). Cells were perfused with Tyrode solution (136 mM NaCl, $5.4 \mathrm{mM} \mathrm{KCl}, 1.0 \mathrm{mM} \mathrm{MgCl} 2,1.8 \mathrm{mM} \mathrm{CaCl} 2,0.33 \mathrm{mM}$ $\mathrm{NaH} 2 \mathrm{PO} 4,10 \mathrm{mM}$ glucose, and $10 \mathrm{mM}$ HEPES; the $\mathrm{pH}$ was adjusted to 7.4 with $\mathrm{NaOH}$.). The cellular electrophysiological parameters were measured in spontaneous beating cardiomyocytes for differentiated cardiomyocytes and neonatal cardiomyocytes, and during pacing with $1 \mathrm{~Hz}$ for adult cardiomyocytes. The nodal, atrial, and ventricular like cardiomyocytes were defined by maximum diastolic membrane potential, action potential amplitude (APA) and the ratio of action potential duration at $90 \%$ to $50 \%$ repolarization (APD90/50) [27]. Both atrial and ventricular cardiomyocytes have lower maximum diastolic membrane potential $(<-50$ $\mathrm{mV}$ ) while nodal is higher than $-50 \mathrm{mV}$. Both atrial and ventricular cardiomyocytes have higher APA (>90 mV) while nodal has lower than $90 \mathrm{mV}$. Atrial cardiomyocytes have a ratio of APD90/APD50 more than 2.0 without plateau phase while ventricular cardiomyocytes have a ratio less than 1.9 with plateau phase.

\section{Calcium Transient}

Calcium transient studies were conducted as in our previous studies [29-32]. In brief, Fluo-4 AM (Invitrogen) was diluted in culture medium and loaded into the cells with a final concentration of $10 \mu \mathrm{M}$ for 30 minutes at room temperature in the dark. Cells were washed with Tyrode $\left(1.8 \mathrm{mM} \mathrm{CaCl}_{2}\right)$ solution and ready for imaging 30 minutes later. The coverslip was transferred into the glass bottom dish for confocal imaging (Corning Incorporated, Corning, NY, USA, https://www.corning. com/). A LSM510 (Zeiss, Oberkochen, Germany, http://www. zeiss.com/) confocal microscope equipped with a $20 \times$ objective was used for calcium transient recording. The Fluo-4 was excited by a $488 \mathrm{~nm}$ line of an Argon laser and emission signals above $505 \mathrm{~nm}$ were recorded. Line scan mode was used for recording the calcium transient at $37^{\circ} \mathrm{C}, 5 \% \mathrm{CO}_{2}$. The $\mathrm{Ca}^{2+}$ level was reported as $\triangle F / F O$, where $F O$ is the resting $\mathrm{Ca}^{2+}$ fluorescence and $\Delta F$ is $F-F O$. $F$ refers to the fluorescence signal recorded at different time points.

\section{Contractility Measurement}

Cell contractility was recorded by MyoCam-S (ION-OPTIX, Westwood, MA, USA, http://www.ionoptix.com/) and IonWizard software (ION-OPTIX) [33]. The coverslip was transferred into a perfusion chamber containing Tyrode buffer $(1.8 \mathrm{mM}$ $\mathrm{CaCl}_{2}$ ) and maintained at $37^{\circ} \mathrm{C}$. The beating cardiomyocyte cell edge was identified by the left and right cursor and located with lonWizard software under the microscope. The cell edge movement was recorded at a magnification of $40 \times$ with the capturing frequency at $250 \mathrm{~Hz}$. Contractility was expressed as a percentage of cell length shortening, calculated with lonWizard software.

\section{Western Blot}

Cells were lysed using RIPA buffer (10 mM Tris- $\mathrm{HCl}(\mathrm{pH} 8.0)$, $1 \mathrm{mM}$ EDTA, 1\% Triton X-100, 0.1\% SDS, $140 \mathrm{mM} \mathrm{NaCl}$ ) with proteinase inhibitor cocktail (Sigma). The lysate was separated with $8 \%$ SDS-PAGE. Membrane was incubated overnight at $4^{\circ} \mathrm{C}$ with rabbit anti- $\beta$-catenin (Abcam 1:1,000), Mesp1
(Abcam, 1:1,000), Isl1 (Abcam, 1:1,000), Nkx2.5 (Santa Cruz, $1: 1,000), \quad \beta$-actin (Abcam, 1:4,000). Goat anti-Rabbit IgG $(\mathrm{H}+\mathrm{L}) / \mathrm{HRP}$ (horseradish peroxidase) (Dako, 1:2000, Glostrup, Denmark, http://www.dako.com/) was used as a secondary antibody and the signal was detected with Clarity ECL Western Blotting Substrate (Bio-Rad, Hercules, CA, USA, http:// www.bio-rad.com/). Band density was measured by Image J (National Institutes of Health, USA, http://www.ncbi.nlm.nih. gov/).

\section{Whole Genome Arrays and Data Analysis}

Gene expression profile was determined on the GeneChip Mouse Gene 2.0 ST Array (Affymetrix, Santa Clara, CA, USA, http://www.affymetrix.com/) at the Genome Center of the University of Hong Kong. Three microgram of total RNA were used for cDNA synthesis, fragmentation and hybridization (Affymetrix). Arrays were scanned with a GeneArray scanner 7G (Affymetrix). Data were background-subtracted and normalized with the Robust Multichip Average method using the software GeneSpring (Agilent Technologies, Santa Clara, CA, USA, https://www.agilent.com/). Expression profiles were established on the basis of duplicate samples collected from independent experiments. Statistical significance was determined with one-way ANOVA test and multiple testing corrections were performed using Benjamini-Hochberg test. $p<0.05$ was considered significant. All samples were compared against the group "Fibroblast" and differentially expressed genes were identified with a fold change cut-off of $>2.0$. Hierarchical clustering was performed with Cluster 3.0 software [34].

\section{Mouse Model of Myocardial Infarction}

$\mathrm{MI}$ and cell transplantation were performed as before [35]. Coronary occlusion was confirmed by the presence of myocardial blanching at the apex. $1 \times 10^{6}$ cells $(20 \mu \mathrm{l}$ in total, diluted in 50\% Matrigel, BD Biosciences) were injected into the infarct border zone (left side, right side, and upside of the infarcted region, three sites in total, $5 \mu \mathrm{l}$ for each site) and the infarcted region (one site in the middle of the infarcted region, $5 \mu \mathrm{l}$ ).

\section{Echocardiography}

Four weeks after transplantation, animals were anesthetized with ketamine $100 \mathrm{mg} / \mathrm{kg}$. The heart was monitored in 2D mode with Vivid q system (GE Healthcare, Little Chalfont, UK, http://www.gehealthcare.com/) with a 11.5-MHz transducer GE 10-RS (GE Healthcare). The image was recorded in 2D mode in the parasternal long axis view. The $M$-mode line passed through the basal segment of the left ventricle between the mitral valve and the papillary muscle. M-mode line was adjusted to be perpendicular to the walls of the left ventricle. Left ventricular ejection fraction was derived from the measurement of LV end-diastolic diameter and LV endsystolic diameter in standard M-mode.

\section{Hemodynamic Assessment}

Hemodynamic assessment was performed as described before $[35,36]$. At 4 weeks after transplantation, the animals were anesthetized with ketamine $100 \mathrm{mg} / \mathrm{kg}$ and xylazine $10 \mathrm{mg} /$ $\mathrm{kg}$. The right carotid artery was gently exposed and isolated. A $1.2-\mathrm{F}, 3.5 \mathrm{~mm}$ electrode spacing rodent pressure-volume catheter (Transonic, Ithaca, NY, USA, http://www.transonic.com/) 
connected to ADVantage Small Animal PV Foundation System (Iworx, Dover, NH, USA, https://www.iworx.com/) was inserted into the artery. Hemodynamic parameters including left ventricular end systolic pressure, maximum increase in left ventricular pressure $(\mathrm{dP} / \mathrm{dt})$ and the slope of end systolic pressure-volume relationship were recorded and analyzed with LabScribe2 (Iworx).

\section{Histological Assessment}

At 4 weeks after transplantation, the animals were sacrificed and the hearts were fixed with $10 \%$ neutral buffered formalin (Sigma), embedded in paraffin. The heart was sectioned (5 $\mu \mathrm{m}$ in thickness) serially from the apex to the base. Every tenth serial section was chosen for further analysis. Sections were used for trichrome staining (ScyTek, Logan, UT, USA, http://www.scytek.com/) or immunohistochemistry [ $\alpha$-Actinin 1:1,000 (Sigma), green florescence protein (GFP) 1:200 (Santa Cruz), vWF 1:200 (Millipore)]. The infarcted wall thickness was measured by the ratio of anterior to septal wall thickness and the percentage of infarction determined by the ratio of blue positive area to whole left ventricle. The engraftment was determined by measuring the GFP positive area and comparing it with the total heart area. The capillary density was measured by counting the VWF positive capillaries per $\mathrm{mm}^{2}$.

\section{DiR Labeling and Cell Tracking}

Cardiospheres were dissociated into single cells by $1 \mathrm{mg} / \mathrm{ml}$ collagenase $B$, followed by $2 \mathrm{mM}$ EDTA treatment. Single cells were labeled with DiR (Life Technologies, Waltham, MA USA, https://www.thermofisher.com/) for 15 minutes and then washed three times with PBS. Labeled cells were transplanted into $\mathrm{MI}$ mice. At different time points following transplantation, the mouse was sacrificed and the heart was dissected out for signal detection using the IVIS Spectrum Preclinical In Vivo Imaging System (PerkinElmer, Waltham , MA, USA, http://www.perkinelmer.com/). The quantity of DiR labelled cells in the heart was analyzed using IVIS Spectrum software.

\section{Statistical Analysis}

Data were analyzed using SPSS software for Windows (IBM, Armonk, NY, USA, http://www.ibm.com/) and are shown as mean \pm SEM (standard error of the mean). Student $t$-test was used for two group comparison and 1-way ANOVA for multiple group comparison with normal data distribution, parametric test, and Turkey Post Hoc tests. A level of $p<0.05$ was considered statistically significant.

\section{RESULTS}

\section{GSK3 $\beta$ Inhibitor-BIO and Oncostatin M Promote Generation of iCS from Mouse Fibroblasts with Somatic Reprogramming}

Mouse embryonic fibroblast cells were infected with retrovirus expressing three transcription factors (Oct4, Sox2, and KIf4). Following overnight infection, the medium was refreshed with Knockout Serum Replacement-based medium that has been proven to be a more efficient system for somatic reprogramming [37]. Quantitative PCR analysis showed that the mRNA level of cardiac progenitor markers Isl1 and Nkx2.5 was significantly upregulated during somatic reprogramming, starting from day 16 (for Is|1) and day 18 (for Nkx2.5) compared with the control green fluorescence protein (GFP) vector at day 6 post retrovirus infection. The mRNA level of Isl1 and Nkx2.5 in cells infected with control GFP remained unchanged at day 22 compared with day 6 (Fig. 1A). The mRNA level of mesoderm marker Bry, another cardiac marker Gata4 and early cardiac progenitor marker Mesp1 were also upregulated compared with the control GFP (Fig. 1B). Nonetheless Mesp1 declined at day 20 compared with day 16 (Fig. 1B). As a result, day 18 post infection was chosen as the experimental time point for the next screening step, when both early (Mesp1) and late (Nkx2.5) cardiac progenitor markers maintained a higher level of expression (Fig. 1A, 1B).

A total of 12 growth factors and chemicals were tested: EGF, bFGF, Wnt3a, BIO, lithium chloride, VEGF, Activin A, HGF, OSM, Interleukin 6, CT-1, and BMP4. Among them, EGF, bFGF, Wnt3a, BIO, and OSM significantly boosted the mRNA level of all Mesp1, Isl1, and Nkx2.5 compared with the basal medium (Fig. 1C). Some other factors also elevated the mRNA level of Isl1 or Nkx2.5 or Isl1 and Nkx2.5, but not Mesp1, and included lithium chloride, VEGF, HGF, CT-1, and BMP4. As BIO and OSM enhanced the mRNA level of IsI1 more than other factors (Fig. 1C), we further determined whether a combination of these two factors could provide an additive effect. Indeed, combining these two factors significantly increased the mRNA level of Mesp1, Isl1, and Nkx2.5 (Fig. 1D). Nonetheless adding a further growth factor into the medium with BIO and OSM did not further increase the same mRNA levels (Fig. 1D).

After plating the cells onto poly-D-lysine coated plates, which were used for cardiosphere formation and isolation [9], cells treated with BIO and OSM, not those untreated, began to aggregate as spheres (Fig. 1E). These spheres, formed during somatic reprogramming with $\mathrm{BIO}$ and OSM treatment, contained Mesp1, Isl1, and Nkx2.5 positive cells (Supporting Information Fig. 1A). They had the capability to form beating cluster of cardiomyocytes after 12 days of differentiation; and the number of beating cluster reached a maximum at 15 days. Those beating cardiomyocytes expressed different cardiomyocytes markers including $\alpha$-Actinin, myosin light chain 2 atrial (atrial specific marker), and myosin light chain 2 ventricular (ventricular specific marker) (Supporting Information Fig. $1 \mathrm{~B}$ and Movie 1). Furthermore, the differentiated cells also contained vWF and $\alpha$-smooth muscle actin positive cells, indicating their endothelium and smooth muscle differentiation capability (Supporting Information Fig. 1C, 1D).

Cell surface marker analysis for cardiospheres revealed that both spheres and no-sphere forming cells derived from reprogramming contained similar high proportion of Sca1 positive and $\mathrm{CD} 105^{\text {high }} \mathrm{CD} 45^{\text {negative }}$ cells as eCS (Supporting Information Fig. 1E). Conversely, spheres forming cells and eCS contained significantly higher population of cKit, Flk1, and CXCR4 positive cells comparing to non-sphere forming cells (Supporting Information Fig. 1E). Moreover, those spheres forming cells were further enriched with cells expressing CKit, Flk1, and CXCR4 on further passage (Supporting Information Fig. 1F). Purified cKit, Flk1, or CXCR4 positive cells from those spheres forming cells had the capability to differentiate into $\alpha$-Actinin positive cardiomyocytes but not those negative populations (Supporting Information Fig. 1G). Furthermore, qPCR analysis revealed that these spheres did 
A

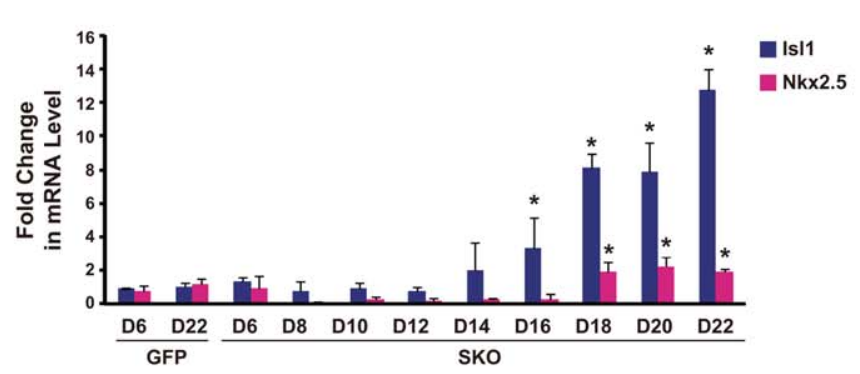

B

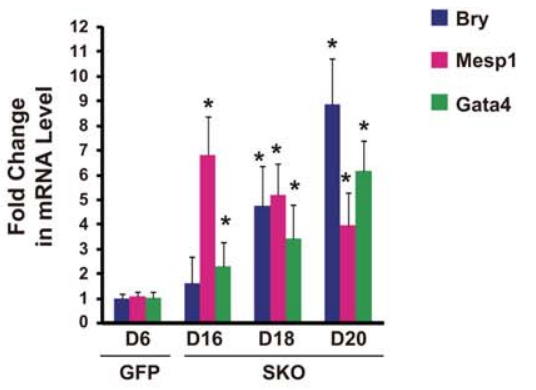

C

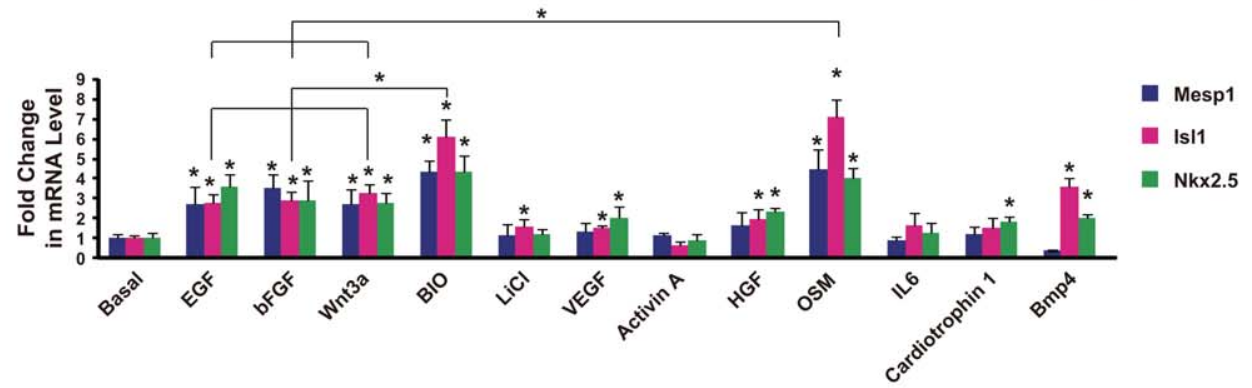

D Mesp1

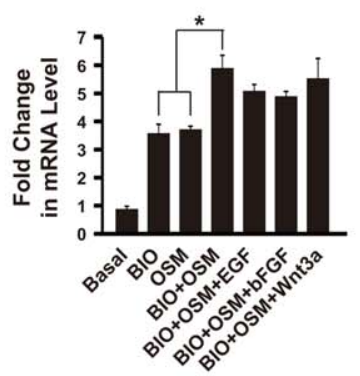

E

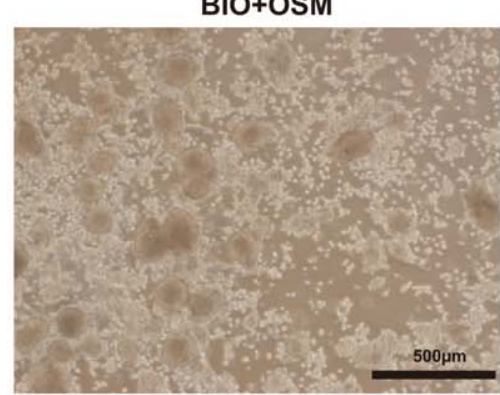

IsI1

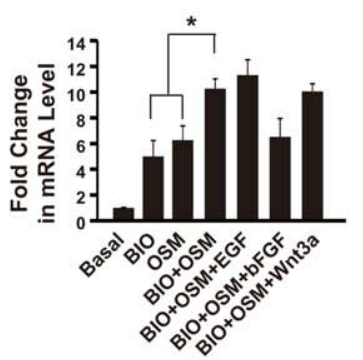

Basal

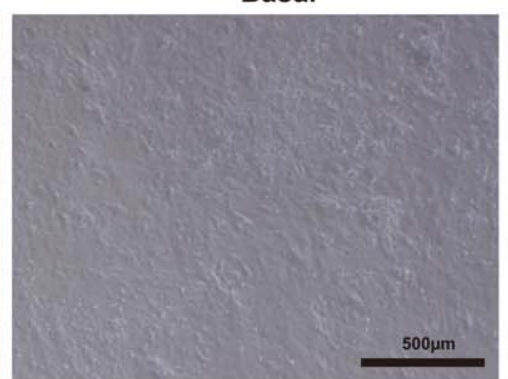

Nkx2.5

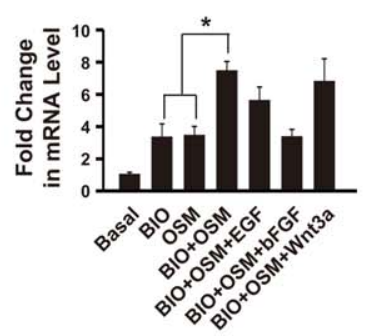

Figure 1. BIO and OSM elevated the mRNA level of cardiac progenitor marker Mesp1, Isl1, and Nkx2.5 and promoted sphere formation in embryonic fibroblasts. (A): qPCR analysis of Isl1 and Nkx2.5 showed that their mRNA level in reproprogramming fibroblasts was overexpressed with SKO (Sox2, Klf4, and Oct4) when the cells were cultured in basal medium compared with GFP from day 6 (D6) to day 22 (D22) during somatic reprogramming $\left(n=3\right.$, biological replicates; ${ }^{*} p<.05$; day time point of interest vs. D6 GFP group); (B) qPCR analysis of mesodermal marker Bry, early cardiac progenitor marker Mesp1 and cardiac marker Gata4 showed that their mRNA level increased from Day 16 (D16) to Day 20 (D20) during reprogramming and Mesp1 tended to decrease from Day 16 to Day20 ( $n=3$, biological replicates; ${ }^{*} p<.05$; day time point of interest vs. D6 GFP group); (C) qPCR analysis of Mesp1, Isl1, and Nkx2.5 for cardiotrophic growth factors or small molecule screening at Day 18 after reprogramming $\left(n=3\right.$, biological replicates; ${ }^{*} p<.05$; treatment group vs. basal group, unless indicated by arrows); (D) qPCR analysis of Mesp1, Isl1, and Nkx2.5 for the combination of BIO and OSM at Day 18 of reprogramming demonstrated their synergistic effects $\left(n=3\right.$, biological replicates; ${ }^{*} p<.05$; indicated by arrows); (E) Sphere formation appeared with BIO and OSM treatment. The figure shows the sphere morphology of induced cardiospheres cultured on poly-D-lysin coated plates after 18 days of reprogramming. No spheres formed in basal meidum without BIO and OSM (middle pannle) after plating onto poly-D-lysin coated plates. Scale bar: $500 \mu \mathrm{m}$. Abbreviations: bFGF, basic fibroblast growth factor; BIO, (2'Z,3'E)- 6-Bromoindirubin$3^{\prime}$-oxime; Bmp4, bone morphogenetic protein 4; CT-1, cardiotrophin-1; EGF, epidermal growth factor; GFP, green fluorescent protein; HGF, hepatocyte growth factor; IL-6, Interleukin 6; OSM, Oncostatin-M; VEGF, vascular endothelial growth factor; Wnt3, Wingless-Type MMTV Integration Site Family, Member 3. 
A

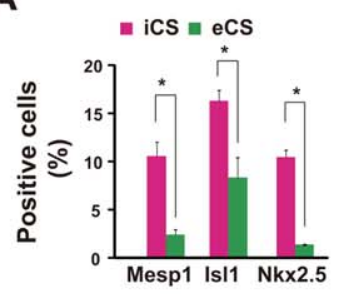

B

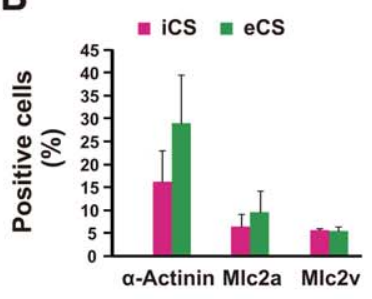

C Nodal-like $=$ Atrial-like $=$ Ventricular-like

D
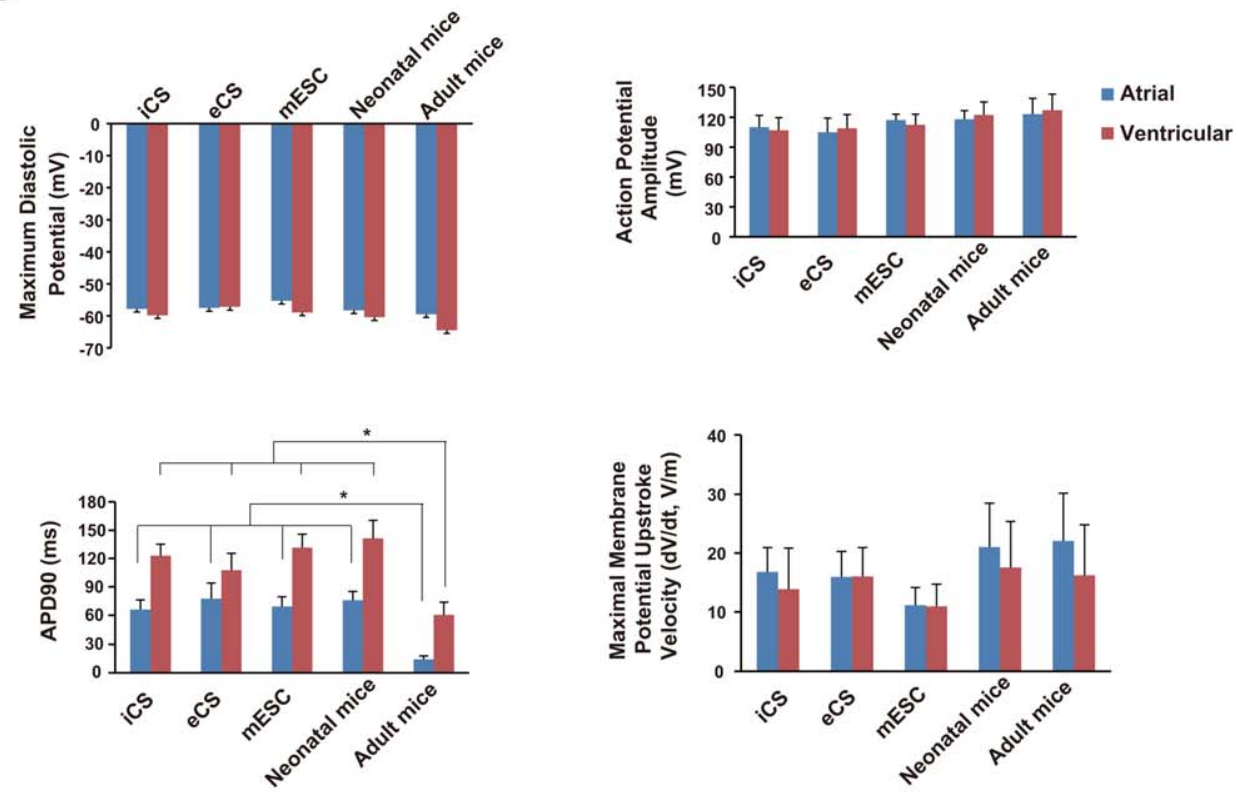

E

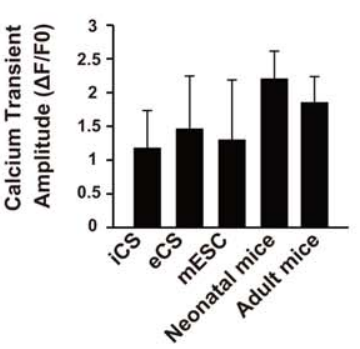

F

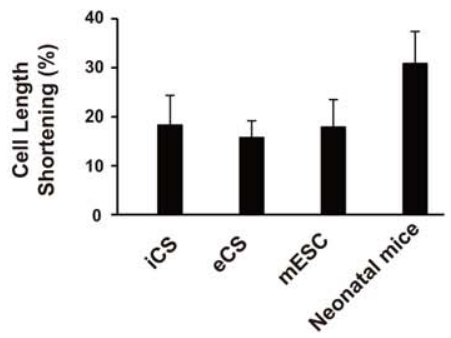

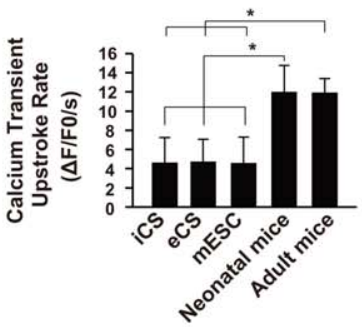

G

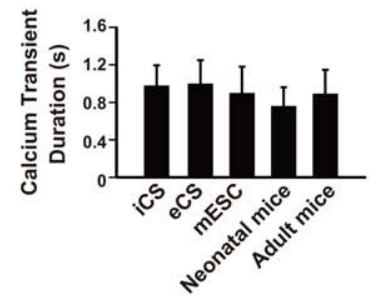

Color Key

Row Z-Score

Figure 2. Functional comparison of iCS generated from adult skin fibroblasts and eCS. (A): iCS contained more Mesp1, Isl1, and Nkx2.5 positive cells than eCS via immunostaining ( $n=3 ;{ }^{*} p<0.05$; iCS vs. eCS) at day 2 after shere formation; (B) After 15 days of cardisphere formation, both iCSs and eCSs had a similar cardiomyocyte differentiation capability shown by measuring $\alpha$-Actinin and Mlc2a and MIc2v positive cell flow cytometry analysis $(n=3)$; (C) Both iCS $(n=63)$, and eCS $(n=50)$ had a similar capability to differentiate into nodal, atrial, and ventricular-like cardiomyocytes as determined by resting membrane potential and action potential (AP) morphology measured by whole cell patch clamp; (D) Cardiomyocytes differentiated from iCSs had similar electrophysiological properties to eCS and mESC- derived cardiomyocytes and neonatal cardiomyocytes from the perspective of maximum diastolic potential, action potential amplitude, APD90, and maximal membrane potential upstroke velocity via patch clamp $(n=55$ for iCS derived cardiomyocytes; $n=42$ for eCS-derived cardiomyocytes; $n=20$ for mESC-derived cardiomyocytes, neonatal and adult cardiomyocytes), ${ }^{*} p<0.05$ as indicated with arrows; (E) Cardiomyocytes differentiated from iCS had similar calcium handling properties to eCS- and mESC-derived cardiomyocytes $(n=10), * p<0.05$ as indicated with arrows; (F) Cardiomyocytes differentiated from iCSs had similar cell contractility to eCS- and mESC-derived cardiomyocytes and neonatal cardiomyocytes $(n=10)$; $(\mathbf{G})$ Whole genome gene expression profile showed iCS clustered with eCS but not fibroblast $(n=2)$. Abbreviations: APD90, action potential duration at $90 \%$ repolarization; eCS, endogenous cardiospheres; iCS, induced cardiospheres; mESC, mouse embryonic stem cell; Mlc2a, myosin light chain 2 atrial; Mlc2v, myosin light chain 2 ventricular. 
not express pluripotent stem cell marker Oct, Sox2, Nanog, or Rex1, confirming that they did not contain pluripotent stem cells. These findings were further confirmed by immune-staining of Nanog in the dissociated spheres (Supporting Information Fig. $1 \mathrm{H}, 1 \mathrm{l})$.

The reprogramming approach using BIO and OSM treatment of mouse embryonic fibroblasts was also applied to adult skin fibroblasts. Similarly, the spheres generated from adult skin fibroblasts contained Mesp1, Isl1, and Nkx2.5 positive cells (Supporting Information Fig. 2) and could differentiate into beating cardiomyocytes (Supporting Information Movie 2). The spheres generated by this approach using somatic reprogramming of pluripotent factors followed by BIO and OSM treatment in either mouse embryonic fibroblasts or adult skin fibroblasts are termed iCS. In general, beating clusters of cardiomyocytes were observed at 32-35 days of culturing from fibroblasts: 18 days for reprogramming and factor induction, 2 days for sphere formation, and 12-15 days for cardiomyocytes differentiation. This reprogramming and differentiation approaches are highly consistent and effective for both mouse embryonic (repeated 13 times) and adult skin fibroblast (repeated 45 times) cells for generation of iCS. In subsequent experiments, iCS generated from adult skin fibroblasts were used for further functional assessment.

\section{Comparison of iCS with eCS and Native Cardiomyocytes}

Compared with eCS isolated from the mouse heart, those iCS derived from adult skin fibroblasts contained a higher percentage of Mesp1, Isl1, and Nkx2.5 positive cells (Fig. 2A and Supporting Information Fig. 2). Nevertheless, both types of cardiospheres showed similar efficacy for in vitro cardiac differentiation when the percentage of $\alpha$-Actinin, myosin light chain 2 atrial, and myosin light chain 2 ventricular positive cells were analyzed (Fig. 2B). Using the patch clamp technique, the action potential was recorded from a single isolated cardiomyocyte to characterize the phenotype of these cells. As defined by the ratio of action potential duration (APD) at $90 \%$ to $50 \%$ repolarization [27], both types of cardiosphere showed a similar level of differentiated nodal-like, atrial-like, and ventricular-like cardiomyocytes (Fig. 2C; Supporting Information Fig. 3).

Next, we further compared the functional phenotypes of cardiomyocytes derived from these two types of cardiospheres (iCS and eCS) and mESC with freshly isolated mouse native cardiomyocytes (neonatal and adult cardiomyocytes). They demonstrated similar maximum diastolic potential, APA, APD at $90 \%$ repolarization and the maximal membrane potential upstroke velocity. Nonetheless the adult cardiomyocytes had a significantly shorter APD at $90 \%$ repolarization compared with other cardiomyocytes (Fig. 2D).

Calcium transient analysis showed that the iCS, eCS, and mESC derived cardiomyocytes had similar level of calcium amplitude and duration of calcium transient. But these differentiated cardiomyocytes had a significantly lower calcium signal upstroke velocity than native cardiomyocytes (Fig. 2E). Assessment of cell contractile function showed that cardiomyocytes from different sources had a similar percentage of cell shortening (Fig. 2F). Our results demonstrated that the in vitro functional parameters of cardiomyocytes derived from eCSs, iCSs, or mESCs were similar; but were less mature compared with native cardiomyocytes. Whole genome expression profile analysis showed that the iCS was clustered with eCS but the fibroblast cells (Fig. 2G), further proving the similarity of iCS and eCS.

\section{Transplantation of iCS Improves Cardiac Function Following Myocardial Infarction}

We investigated the therapeutic effects of transplanting iCS versus eCS into an animal model of acute $\mathrm{MI}$ in immunodeficient mice (NOD/SCID). Compared with phosphate-buffered saline or the iCS parental fibroblast cell injection, both iCS and eCS transplantation significantly improved left ventricular ejection fraction at week 4 after cell transplantation as determined by serial echocardiogram (Fig. 3A, 3B). Invasive hemodynamic assessment at week 4 also confirmed that compared with saline or fibroblast injection, both iCS and eCS transplantation improved left ventricular end systolic pressure, maximum increase in left ventricular pressure and the slope of end systolic pressure-volume relationship (Fig. 3C, 3D).

Histological examination with Trichrome staining showed that iCS and eCS transplantation significantly increased anterior/septal ventricular wall thickness and reduced infarct size (Fig. 3E--3G). In vivo tracking of the transplanted cells using DiR labeling revealed that transplanted iCSs and eCSs were engrafted into the myocardium at a similar level and remained detectable 12 weeks after transplantation. Nevertheless, the average radiant efficiency of DiR was significantly reduced at week 4 compared with 5 minutes after cell transplantation (week 0 ) and this was further reduced at week 12 (Fig. 4A, 4B).

To further determine the fate of the transplanted cells, they were labeled with lentivirus expressing GFP and then transplanted into MI mice. The GFP positive cells were engrafted in the mouse heart at a similar level and double staining for GFP and $\alpha$-Actinin showed that the transplanted iCS and eCS had the potential for in vivo differentiation into cardiomyocytes 4 weeks after cell transplantation (Fig. 4C, 4D).

In addition, transplantation of both iCS and eCS significantly increased the capillary density as determined by vWF staining of the peri-infarct region and infarcted region compared with saline injection (Fig. 4E, 4F). In addition, double staining of GFP and VWF revealed potential endothelial cell differentiation of transplanted iCS or eCS (Supporting Information Fig. 4). In summary, iCS have similar therapeutic effects to eCS and improve cardiac function following MI. Of note, no tumor formation was observed 12 weeks following transplantation.

\section{Induction of Cardiosphere Formation from Fibroblasts by BIO and OSM via $\beta$-Catenin}

$\beta$-catenin is stabilized by the Gsk3 $\beta$ inhibitor-BIO and is critical for the maintenance of cardiac progenitor cells [38, 39]. Accordingly, we hypothesized that BIO and OSM induced cardiosphere formation via upregulation of $\beta$-catenin. Indeed, both $\mathrm{BIO}$ and OSM alone or in combination increased the protein level of $\beta$-catenin as well as the expression of Mesp1, Isl1, and Nkx2.5 when compared with basal medium without BIO and OSM (Fig. 5A). Knocking down $\beta$-catenin through shRNA decreased the protein level of Mesp1, Isl1, and Nkx2.5 compared with cells infected with scramble shRNA (Fig. 5B), 
Pre-MI

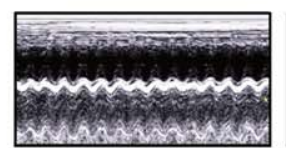

MI+Saline

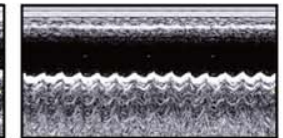

Ml+Fibroblast
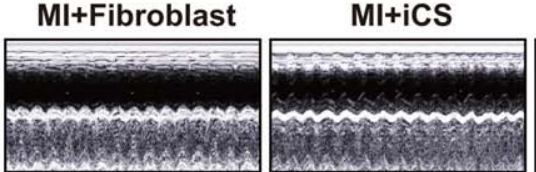

$\mathrm{Ml}+\mathrm{eCS}$

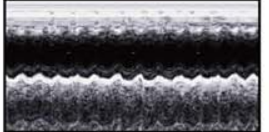

C

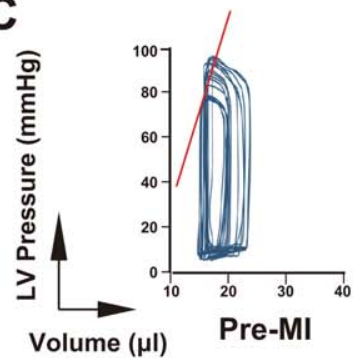

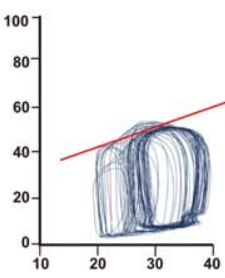

MI+Saline
Ml+Fibroblast
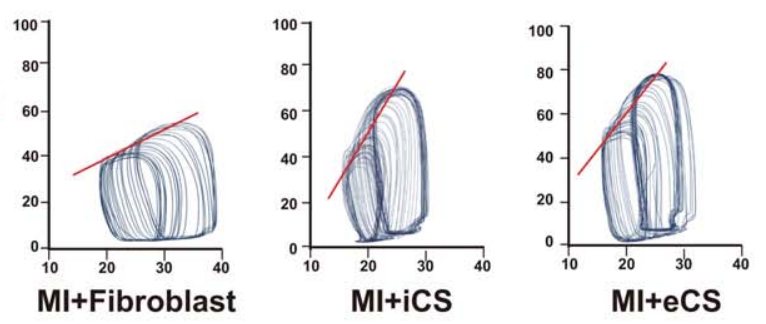

D

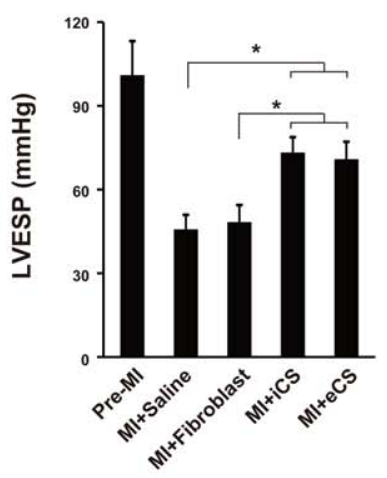

E

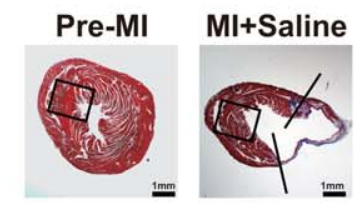

$\mathrm{Ml+}$

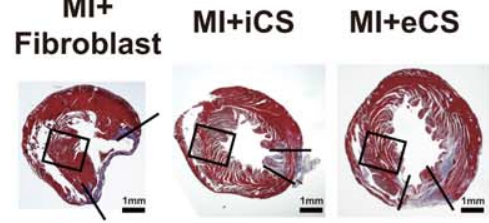

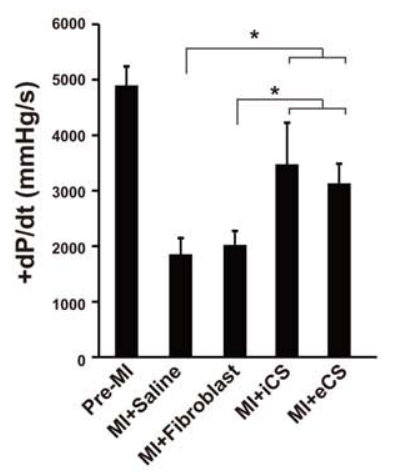

F
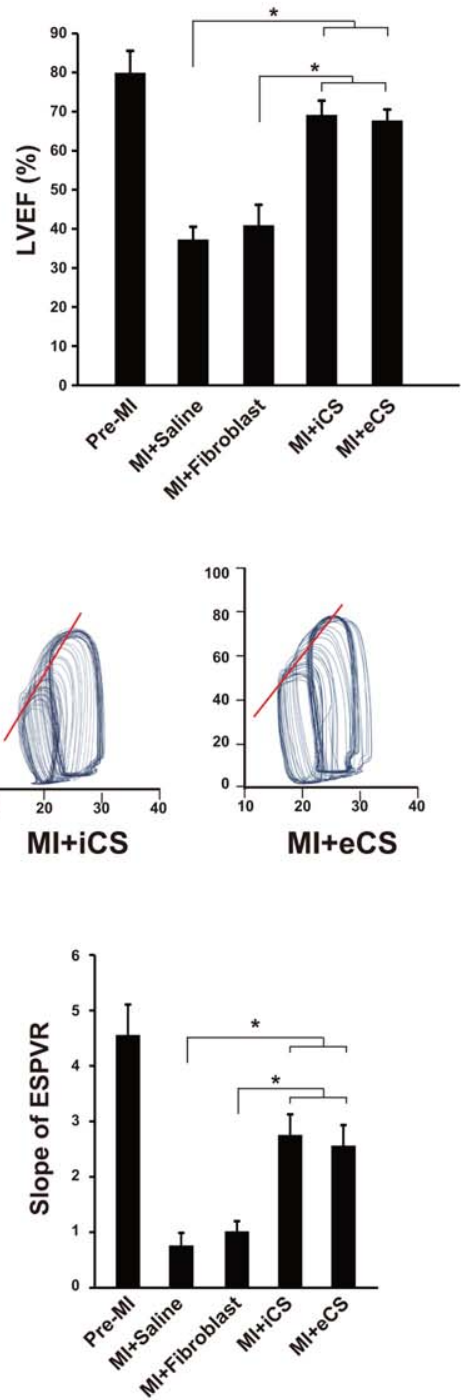

G

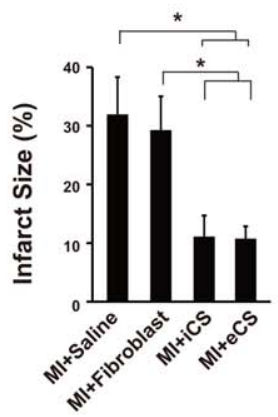

Figure 3. Transplantation of iCS improves cardiac function as eCS following MI. (A): Representative M-mode echocardiographic tracing at week 4 after MI induction and cardiosphere transplantation showed cardiac function improvement; (B) Assessment of LVEF by echocardiogram at week 4 after cell transplantation showed both iCSs and eCSs achieved a similar level of cardiac function improvement $(n=8) ; * p<0.05$; as indicated by arrows; (C) Representative tracing of the pressure-volume loop assessment to measure slope of ESPVR at week 4 after cell transplantation showing cardiac function improvement; (D) Hemodynamic assessment at week 4 after cell transplantation showed both iCS and eCS achieved a similar level of cardiac function improvement $(n=8)$; ${ }^{*} p<0.05$; as indicated by arrows; (E) Trichrome staining of paraffin embedded heart sections at week 4 after cell transplantation indicated wall thickness increase and infarct size decrease. The blue region between the dark lines indicates the infarcted region; the black frame indicates the region (middle of the septum) for septal thickness measurement; Scale bar: $1 \mathrm{~mm}$. (F): Left ventricular wall thickness was measured by the ratio of anterior to septal wall thickness at week 4 after cell transplantation and indicated that both iCS and eCS had a similar increase in wall thickness $(n=8) ;{ }^{*} p<0.05$; as indicated by arrows; $(\mathbf{G})$ Infarct size measurement by the percentage area with collagen deposit using trichrome staining at week 4 after cell transplantation indicated both iCS and eCS achieved a similar decrease in infarct size $(n=8)$; ${ }^{*} p<0.05$; as indicated by arrows. Abbreviations: $+\mathrm{dP} / \mathrm{dt}$, peak rate of pressure rise; eCS, endogenous cardiospheres; ESPVR, end systolic pressure-volume relationship; iCS, induced cardiospheres; LV, left ventricular; LVEF = left ventricular ejection fraction; LVESP, left ventricular end systolic pressure; MI, myocardial infarction; Pre-MI, before myocardial infarction. 
A

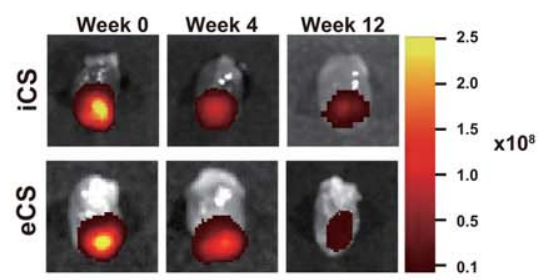

B

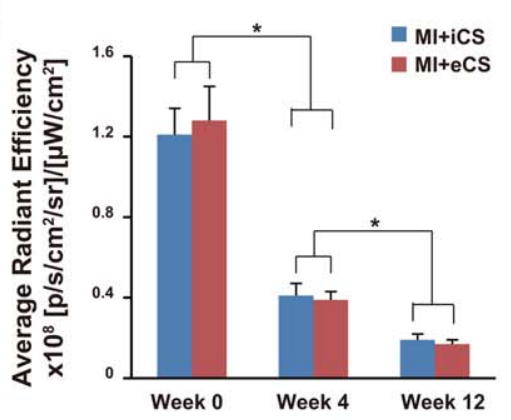

C
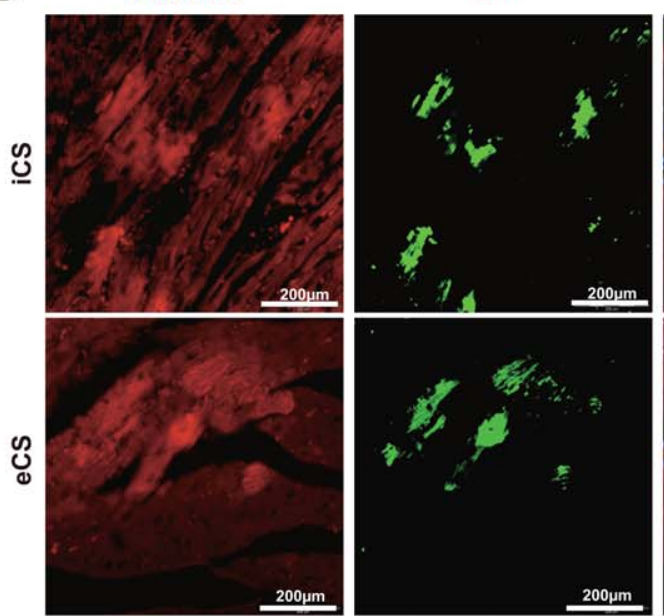

E

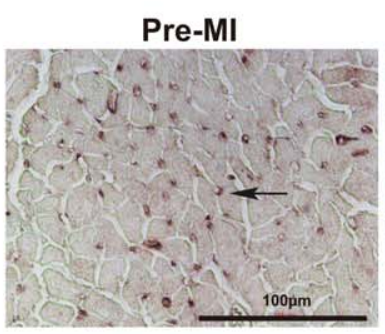

MI +eCS

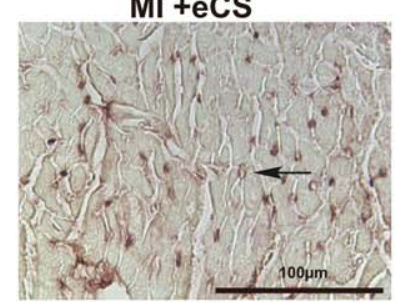

MI+Saline

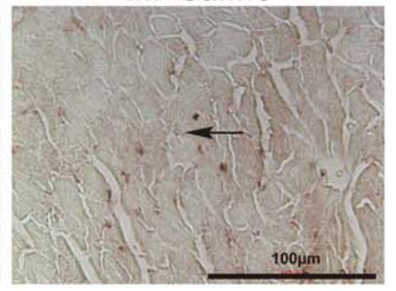

$\mathrm{MI}+\mathrm{iCS}$

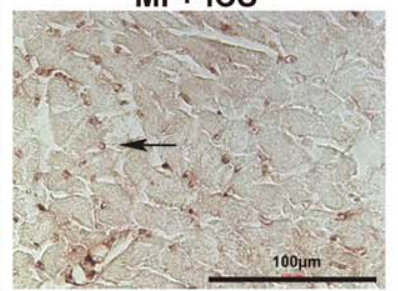

Merge/DAPI
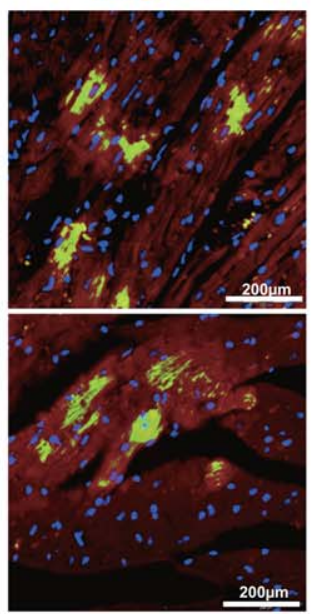
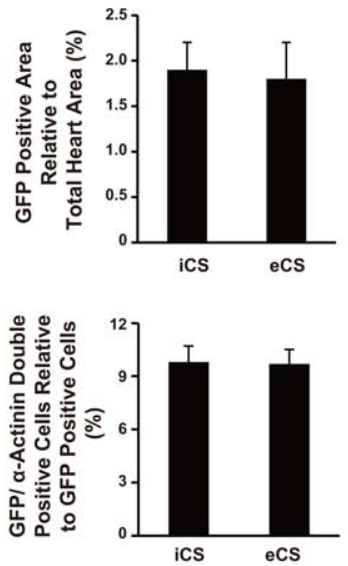

F

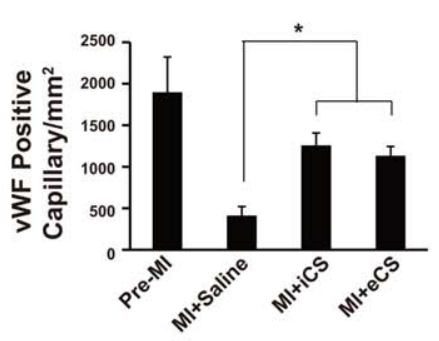

Infarcted Region

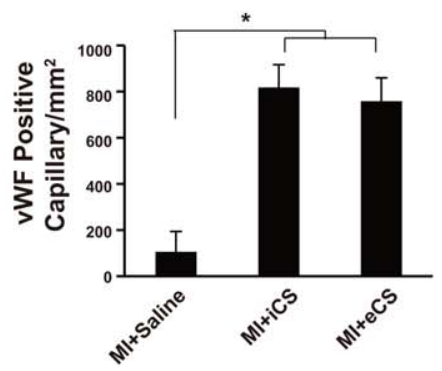

Figure 4. Transplanted iCS participate in cardiac regeneration in vivo and promote angiogenesis as eCS. (A): Representative figures of DiR labeled cells in the heart at week 0 ( 5 minutes after cell transplantation), week 4 and week 12 after cell transplantation in a mouse model of myocardial infarction, heatmap showed the average radiant efficiency; (B) Average radiant efficiency of DiR labeled cells in the heart after cell transplantation indicated both iCS and eCS achieved similar engraftment and were still detectable at week $12(n=8)$; ${ }^{*} p<0.05$; as indicated by arrows; (C) Immunostaining of paraffin embedded heart sections at week 4 after cell transplantation with $\alpha$ Actinin and GFP which indicates that the transplanted cardiospheres participated in cardiac regeneration; Scale bar: $200 \mu \mathrm{m}$. (D): Cell engraftment measured by immunohistochemistry showed both iCS and eCS achieved a similar level of cell engraftment (up panel, $n=8$ ). (E): VWF staining of paraffin embedded heart sections showed that capillary density increased after transplantation of iCS and eCS, dark arrow indicates the vWF positive capillary; Scale bar: $100 \mu \mathrm{m}$. (F): Peri-infarct and infarcted region capillary number counting showed both iCS and eCS increased capillary density to a similar degree $\left(n=8 ;{ }^{*} p<0.05\right.$; as indicated by arrows). Abbreviations: eCS, endogenous cardiospheres; GFP, green fluorescent protein; iCS, induced cardiospheres; MI, myocardial infarction; Pre-MI, before myocardial infarction; vWF, von Willebrand Factor. 


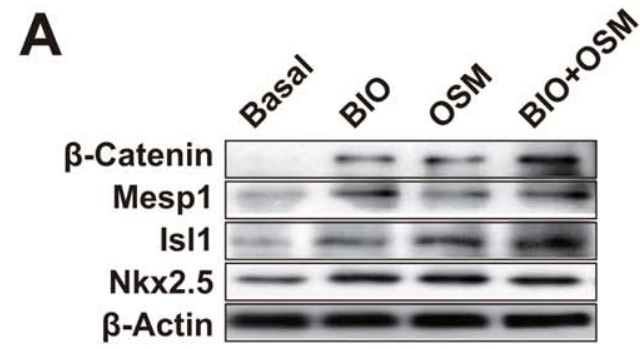

B

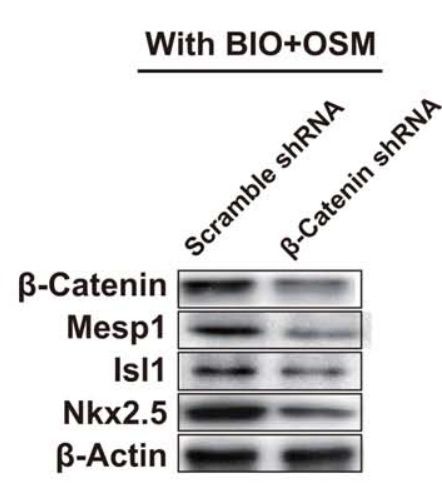

B-Actin
$\beta$-Actin
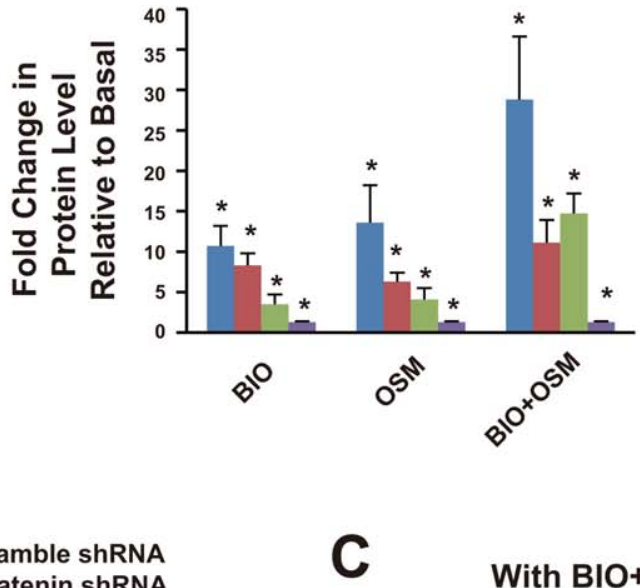

- $\beta$-Catenin

- Mesp1

Is|1

Nkx2.5
- Scramble shRNA

$\beta$-Catenin shRNA

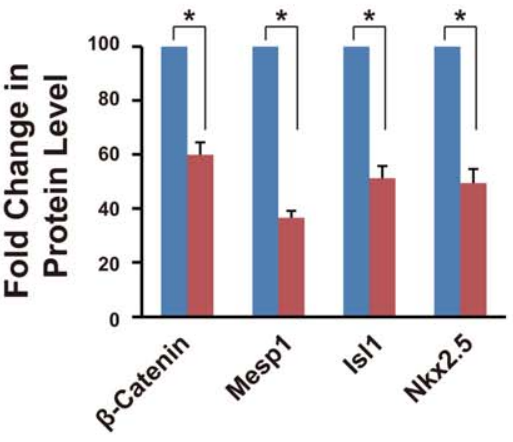

With BIO+OSM

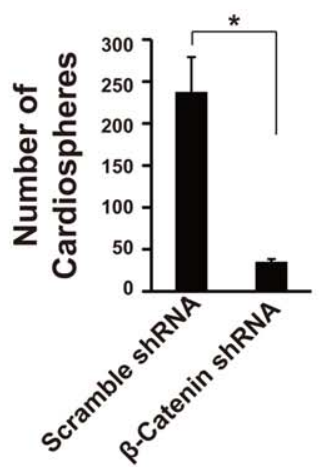

E

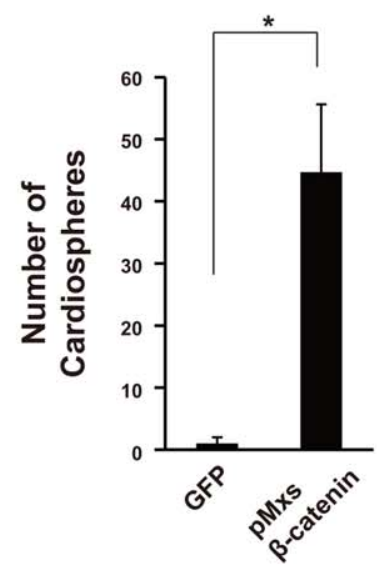

Figure 5. The BIO and OSM induced cardiosphere (iCS) generation through $\beta$-catenin pathway in adult skin fibroblasts infected with SKO (Sox2, Klf4, and Oct4) treated with BIO or OSM or BIO and OSM. (A): Western blot of $\beta$-catenin, Mesp1, Isl1, and Nkx2.5 at day 15 after iCS formation. Representative immunoblot (left panel) and band density measurement (right panel) showed the $\beta$-catenin level was elevated by BIO and OSM stimulation, similar to Mesp1, IsI1, and Nkx2.5 $\left(n=3 ;{ }^{*} p<0.05\right.$; groups with growth factors vs. basal group); Basal: medium without BIO and OSM. (B): Representative immunoblot (left panel) and band density measurement (right panel) showed $\beta$-catenin level was decreased by shRNA knock down, with corresponding supression of Mesp1, Isl1, and Nkx2.5 expression $\left(n=3\right.$; ${ }^{*} p<0.05$; sh-catenin vs. scramble group); (C) Sphere number count after knocking down $\beta$-catenin showed that iCS formation decreased with knock down of $\beta$-catenin level in the presence of BIO and OSM $(n=3 ; * p<0.05$; sh-catenin vs. scramble group); (D) Representative western blot (left panel) and band density measurement (right panel) indicated overexpression of $\beta$-catenin in the absence of BIO and OSM induced $\beta$-catenin that promotes Mesp1, Isl1, and Nkx2.5 expression $\left(n=3{ }^{*} p<0.05\right.$; pMXs- $\beta$-catenin vs. GFP group); (E) Cardiosphere number count after overexpressing $\beta$-catenin without BIO and OSM treatment showed $\beta$-catenin could promote iCS formation $\left(n=3{ }^{*} p<0.05\right.$; pMXs- $\beta$-catenin overexpression vs. GFP group). Abbreviations: BIO, $\left(2^{\prime} Z, 3^{\prime} E\right)$ - 6-Bromoindirubin-3'oxime; GFP, green fluoresent protein; OSM, Oncostatin-M. 
and reduced cardiosphere formation (Fig. 5C). Nonetheless overexpressing $\beta$-catenin upregulated the protein level of Mesp1, Isl1, and Nkx2.5 compared with GFP (Fig. 5D), and promoted the formation of cardiospheres in the absence of $\mathrm{BIO}$ and OSM (Fig. 5E). These findings confirm the critical role of $\beta$-catenin in the induction of cardiosphere formation from fibroblasts.

\section{Induction of Cardiosphere Formation from Human Fibroblasts}

We further attempted the generation of iCS from human skin fibroblast. Human iCS could be successfully generated via the same reprogramming approach as the mouse skin fibroblast (Supporting Information Fig. 5A). Those cells derived from the human iCS were positive for cardiac progenitor cell markers Mesp1, Isl1, and Nkx2.5 (Supporting Information Fig. 5B). While the differentiated cardiomyocytes were positive for $\alpha$ Actinin, Mlc-2a and Mlc-2v (Supporting Information Fig. 5C), no spontaneously beating could be observed as previous reported in human eCS $[8,9]$. Nevertheless, those differentiated cardiomyocytes had functional calcium transient activity and responded to caffeine stimulation (Supporting Information Fig. 5D).

\section{Discussion}

Cardiac progenitor cells have been proposed as a novel therapy for the treatment of MI. Cardiospheres are a type of cardiac progenitor cell that is potentially safe and effective for cardiac regeneration following $\mathrm{MI}$ in both animal [40] and human [20] studies. Nevertheless, the widespread clinical application of autologous eCS is limited by the need for invasive harvesting, and their number, proliferation and differentiation ability decline during the aging process [17, 19, 40, 41]. Direct reprogramming of fibroblasts into cardiomyocytes is another promising approach to achieve cardiac regeneration but is limited by the low conversion rate and the potential arrhythmia risk associated with cardiomyocyte transplantation. We present a new strategy to generate iCS from adult mouse skin fibroblasts using a cocktail approach with expression of pluripotent stem cell factors and combination of Gsk3 $\beta$ inhibitor-BIO and OSM. Furthermore, human iCS can be generated using similar approach. Our results suggest that these cardiotrophic growth factors induce cardiosphere formation via upregulation of $\beta$-catenin. These iCS are capable of differentiation into functional cardiomyocytes in vitro, from the perspective of action potential and calcium transient, and incorporate into the myocardium in a MI model as eCS with subsequent cardiac function improvement. This study provides the first proof-of-principle results that demonstrate the feasibility of generating donor-specific iCS via a somatic reprogramming process, providing a new source of cell therapy for treatment of MI.

First, we used the expression of a panel of cardiac transcription factors including Mesp1, Is|1, and Nkx2.5 to screen for the optimal cocktail of cardiotrophic growth factors for iCS formation after reprogramming with pluripotent stem cell factors. These transcription factors have been shown via lineage tracing studies to be important for heart development and maintenance of cardiac progenitor cells [13-15]. Quantitative real-time PCR analysis showed that all these markers were upregulated during somatic reprogramming and were further enhanced after adding two more cardiotrophic factors, Gsk3 $\beta$ inhibitor-BIO and OSM. Sphere formation could be observed after seeding the cells onto poly-D-lysine coated plates as described in the isolation of eCS $[8,9,19]$. These iCS generated from this somatic reprogramming approach were positive for all three cardiac progenitor markers Mesp1, Is|1, and $\mathrm{Nkx2.5}$ and had the capability to differentiate into functional cardiomyocytes. The iCSs contained only a different proportion of Mesp1, Isl1, and Nkx2.5 positive cells, indicating that they were a mixed cell population, in accordance with previous reports $[9,19,40]$.

The cardiomyocytes derived from the iCS generated from adult skin fibroblasts were comparable with those derived from eCS and mESC in terms of cellular electrophysiology, calcium handling, and contractile function. Moreover, transplantation of the iCS following MI provided similar improvement to that of eCS in terms of cardiac function, reduction in infarct size and increased capillary density, consistent with previous findings in eCS [7].

Our results also provide novel insight into the mechanism of cardiosphere formation. $\beta$-catenin is an important regulator of cardiac progenitor cell fate maintenance, proliferation and cardiomyocyte differentiation [42]. It directly regulates isl1 expression [43] and loss of $\beta$-catenin decreases the cardiac progenitor population and leads to failure in heart development [44]. It is well known that Gsk3 $\beta$ inhibitor-BIO is a stabilizer of $\beta$-catenin that is critical for heart development $[38,39]$. While the action of OSM on $\beta$-catenin is unknown, prior studies have shown that it promotes cardiomyocyte dedifferentiation and cardiac progenitor cell proliferation in both $\mathrm{MI}$ and heart failure [45]. Here, we demonstrated the pivotal role of $\beta$-catenin in the formation of iCS. Both BIO and OSM significantly increased the protein level of $\beta$ catenin. Manipulating the expression of $\beta$-catenin during the formation of iCS significantly affected the efficiency of generation of iCS.

This study had several limitations. First, the use of retrovirus vector may limit the clinical application of this approach. Whether the use of other nonintegrated vectors or small molecules for reprogramming can provide similar efficacy remains unclear. Second, the iCS contains a mixed progenitor cell population as in the eCS. Therefore, the contribution of different types of progenitor cells in iCS to cardiac differentiation and regeneration needs to be further investigated. Third, the longterm safety and efficacy of iCS transplantation are unknown. Although we did not observe any tumor formation after 3 months, the proarrhythmic risk of iCS transplantation was not addressed in this study and their long-term therapeutic efficacy is unclear. Finally, the low retention of transplanted cells hinders the potential applications, which is one of the most important issues should be addressed in the cell therapy field for MI.

\section{CONCLUSION}

iCS generated from adult skin fibroblasts by somatic reprogramming and a cocktail of Gsk3 $\beta$ inhibitor-BIO and OSM may represent a novel source for cell therapy in MI. 


\section{ACKNOWLEDGMENTS}

This work was supported by the Hong Kong Research Grant Council: Theme Based Research Scheme (T12-705/11 to H.F.T.); Strategic Priority Research Program of the Chinese Academy of Sciences (XDA01020106, to M.A.E.).

\section{AUtHOR CONTRIBUtions}

J.-Y.X.: conception and design, provision of study material, collection and assembly of data, data analysis and interpretation, manuscript writing; Y.-K.L.: manuscript writing; X.R.: administrative support, provision of study material; S.-Y.L.: data analysis and interpretation; J.Y.: administrative support; K.-W.A.: administrative support, provision of study material; W.-H.L.: administrative support, provision of study material; M.A.E.: manuscript writing; H.-F.T.: conception and design, data analysis and interpretation, manuscript writing, final approval of manuscript.

\section{Disclosure of POTENTIAL CONFLicts OF INTERESt}

The authors indicate no potential conflicts of interest.

\section{REFERENCES}

1 Lozano R, Naghavi $M$, Foreman $K$ et al. Global and regional mortality from 235 causes of death for 20 age groups in 1990 and 2010: A systematic analysis for the global burden of disease study 2010. Lancet 2012;380:2095-2128

2 Murray CJ, Vos T, Lozano R et al. Disability-adjusted life years (DALYs) for 291 diseases and injuries in 21 regions, 1990-2010: A systematic analysis for the global burden of disease study 2010. Lancet 2012;380: 2197-2223.

3 Kawamoto A, Gwon $\mathrm{HC}$, Iwaguro $\mathrm{H}$ et al Therapeutic potential of ex vivo expanded endothelial progenitor cells for myocardial ischemia. Circulation 2001;103:634-637.

4 Siu CW Tse HF. Cardiac regeneration: Messages from CADUCEUS. Lancet 2012;379: 870-871.

5 Sanganalmath SK Bolli R. Cell therapy for heart failure: A comprehensive overview of experimental and clinical studies, current challenges, and future directions. Circ Res 2013;113:810-834.

6 Tse HF, Siu CW, Zhu SG et al. Paracrine effects of direct intramyocardial implantation of bone marrow derived cells to enhance neovascularization in chronic ischaemic myocardium. Eur J Heart Fail 2007;9:747-753.

7 Fazel S, Cimini M, Chen L et al. Cardioprotective c-kit + cells are from the bone marrow and regulate the myocardial balance of angiogenic cytokines. J Clin Invest 2006; 116:1865-1877.

8 Messina E, De Angelis L, Frati G et al. Isolation and expansion of adult cardiac stem cells from human and murine heart. Circ Res 2004;95:911-921.

9 Smith RR, Barile L, Cho HC et al. Regenerative potential of cardiosphere-derived cells expanded from percutaneous endomyocardial biopsy specimens. Circulation 2007;115:896-908. 10 Beltrami AP, Barlucchi L, Torella D et al. Adult cardiac stem cells are multipotent and support myocardial regeneration. Cell 2003; 114:763-776.

11 Kattman SJ, Huber TL Keller GM. Multipotent flk-1+ cardiovascular progenitor cells give rise to the cardiomyocyte, endothelial, and vascular smooth muscle lineages. Dev Cell 2006;11:723-732.
12 Zhou B, Ma Q, Rajagopal S et al. Epicardial progenitors contribute to the cardiomyocyte lineage in the developing heart. Nature 2008;454:109-113.

13 Bondue A, Lapouge G, Paulissen C et al. Mesp1 acts as a master regulator of multipotent cardiovascular progenitor specification. Cell Stem Cell 2008;3:69-84.

14 Cai $C L$, Liang $X$, Shi $Y$ et al. Isl1 identifies a cardiac progenitor population that proliferates prior to differentiation and contributes a majority of cells to the heart. Dev Cell 2003;5:877-889.

15 Wu SM, Fujiwara Y, Cibulsky SM et al. Developmental origin of a bipotential myocardial and smooth muscle cell precursor in the mammalian heart. Cell 2006;127:11371150.

16 Oyama T, Nagai T, Wada $\mathrm{H}$ et al. Cardiac side population cells have a potential to migrate and differentiate into cardiomyocytes in vitro and in vivo. J Cell Biol 2007;176:329341.

17 Kucia M, Dawn B, Hunt $G$ et al. Cells expressing early cardiac markers reside in the bone marrow and are mobilized into the peripheral blood after myocardial infarction. Circ Res 2004;95:1191-1199.

18 Weil BR, Suzuki G, Leiker MM et al. Comparative efficacy of intracoronary alloge neic mesenchymal stem cells and cardiosphere-derived cells in swine with hibernating myocardium. Circ Res 2015;117: 634-644.

19 Mishra R, Vijayan K, Colletti EJ et al. Characterization and functionality of cardiac progenitor cells in congenital heart patients. Circulation 2011;123:364-373.

20 Ishigami S, Ohtsuki S, Tarui S et al. Intracoronary autologous cardiac progenitor cell transfer in patients with hypoplastic left heart syndrome (TICAP): A prospective phase 1 controlled Trial. Circ Res 2013;116:653664.

21 Menasche $P$, Vanneaux $V$, Fabreguettes JR et al. Towards a clinical use of human embryonic stem cell-derived cardiac progenitors: A translational experience. Eur Heart J 2015;36:743-750.

22 Efe JA, Hilcove S, Kim J et al. Conversion of mouse fibroblasts into cardiomyocytes using a direct reprogramming strategy. Nat Cell Biol 2011;13:215-222.
23 Wang $\mathrm{H}$, Cao $\mathrm{N}$, Spencer $\mathrm{Cl}$ et al. Small molecules enable cardiac reprogramming of mouse fibroblasts with a single factor, Oct4. Cell Rep 2014;6:951-960.

24 Li D, Wu J, Bai Y et al. Isolation and culture of adult mouse cardiomyocytes for cell signaling and in vitro cardiac hypertrophy. J Vis Exp 2014;(87).

25 Takahashi K Yamanaka S. Induction of pluripotent stem cells from mouse embryonic and adult fibroblast cultures by defined factors. Cell 2006;126:663-676.

26 Naviaux RK, Costanzi E, Haas $M$ et al. The $\mathrm{pCL}$ vector system: Rapid production of helper-free, high-titer, recombinant retroviruses. J Virol 1996;70:5701-5705.

27 Kuzmenkin A, Liang H, Xu G et al. Functional characterization of cardiomyocytes derived from murine induced pluripotent stem cells in vitro. FASEB J 2009;23:41684180 .

28 Karmazinova M Lacinova L. Measurement of cellular excitability by whole cell patch clamp technique. Physiol Res 2010;59: S1-S7.

29 Guatimosim S, Guatimosim C Song LS. Imaging calcium sparks in cardiac myocytes. Methods Mol Biol 2011;689:205-214.

30 Lee YK, Ho PW, Schick R et al. Modeling of friedreich ataxia-related iron overloading cardiomyopathy using patient-specificinduced pluripotent stem cells. Pflugers Arch 2014;466:1831-1844.

31 Lee YK, Ng KM, Lai WH et al. Calcium homeostasis in human induced pluripotent stem cell-derived cardiomyocytes. Stem Cell Rev 2011;7:976-986.

$32 \mathrm{Ng} \mathrm{K}$, Lee $\mathrm{Y}$, Lai $\mathrm{W}$ et al. Exogenous expression of human apoA-I enhances cardiac differentiation of pluripotent stem Cells. Plos One 2011;6:e19787.

33 Pointon A, Harmer AR, Dale IL et al. Assessment of cardiomyocyte contraction in human-induced pluripotent stem cell-derived cardiomyocytes. Toxicol Sci 2015;144:227237.

34 de Hoon MJ, Imoto S, Nolan J et al. Open source clustering software. Bioinformatics 2004;20:1453-1454.

35 Liao SY, Liu Y, Siu CW et al. Proarrhythmic risk of embryonic stem cell-derived cardiomyocyte transplantation in infarcted myocardium. Heart Rhythm 2010;7:1852-1859. 
36 Pacher $\mathrm{P}$, Nagayama T, Mukhopadhyay $\mathrm{P}$ et al. Measurement of cardiac function using pressure-volume conductance catheter technique in mice and rats. Nat Protoc 2008;3: 1422-1434.

37 Chen J, Liu J, Han Q et al. Towards an optimized culture medium for the generation of mouse induced pluripotent stem cells. J Biol Chem 2010;285:31066-31072.

38 Kwon C, Arnold J, Hsiao EC et al. Canonical Wnt signaling is a positive regulator of mammalian cardiac progenitors. Proc Natl Acad Sci USA 2007;104:10894-10899.

39 Qyang $\mathrm{Y}$, Martin-Puig S, Chiravuri M et al. The renewal and differentiation of
Isl1+ cardiovascular progenitors are controlled by a Wnt/beta-catenin pathway. Cell Stem Cell 2007;1:165-179.

40 Simpson DL, Mishra R, Sharma S et al. A strong regenerative ability of cardiac stem cells derived from neonatal hearts. Circulation 2012;126:S46-S53.

41 Torella $D$, Rota $M$, Nurzynska $D$ et al. Cardiac stem cell and myocyte aging, heart failure, and insulin-like growth factor-1 overexpression. Circ Res 2004;94:514-524.

42 Gessert S Kuhl M. The multiple phases and faces of Wnt signaling during cardiac differentiation and development. Circ Res 2010; 107:186-199.
43 Lin L, Cui L, Zhou W et al. Beta-catenin directly regulates Islet1 expression in cardiovascular progenitors and is required for multiple aspects of cardiogenesis. Proc Natl Acad Sci USA 2007;104:9313-9318.

44 Cohen ED, Wang Z, Lepore JJ et al. Wnt/ beta-catenin signaling promotes expansion of Isl-1-positive cardiac progenitor cells through regulation of FGF signaling. J Clin Invest 2007;117:1794-1804.

45 Kubin T, Poling J, Kostin S et al. Oncostatin $\mathrm{M}$ is a major mediator of cardiomyocyte dedifferentiation and remodeling. Cell Stem Cell 2011;9:420-432.

See www.StemCells.com for supporting information available online. 